\title{
OPEN Serum fibroblast growth factor 21 levels after out of hospital cardiac arrest are associated with neurological outcome
}

Pirkka T. Pekkarinen ${ }^{1 凶}$, Markus B. Skrifvars², Ville Lievonen², Pekka Jakkula, Laura Albrecht ${ }^{2}$, Pekka Loisa ${ }^{3}$, Marjaana Tiainen ${ }^{4}$, Ville Pettilä $^{1}$, Matti Reinikainen ${ }^{5}$ \& Johanna Hästbacka ${ }^{1}$

Fibroblast growth factor (FGF) 21 is a marker associated with mitochondrial and cellular stress. Cardiac arrest causes mitochondrial stress, and we tested if FGF 21 would reflect the severity of hypoxiareperfusion injury after cardiac arrest. We measured serum concentrations of FGF 21 in 112 patients on ICU admission and 24, 48 and $72 \mathrm{~h}$ after out-of-hospital cardiac arrest with shockable initial rhythm included in the COMACARE study (NCT02698917). All patients received targeted temperature management for $24 \mathrm{~h}$. We defined 6-month cerebral performance category 1-2 as good and 3-5 as poor neurological outcome. We used samples from 40 non-critically ill emergency room patients as controls. We assessed group differences with the Mann Whitney $U$ test and temporal differences with linear modeling with restricted maximum likelihood estimation. We used multivariate logistic regression to assess the independent predictive value of FGF 21 concentration for neurologic outcome. The median (inter-quartile range, IOR) FGF 21 concentration was $0.25(0.094-0.91) \mathrm{ng} / \mathrm{ml}$ in controls, $0.79(0.37-1.6) \mathrm{ng} / \mathrm{ml}$ in patients at ICU admission $(P<0.001$ compared to controls) and peaked at $48 \mathrm{~h}[1.2(0.46-2.5) \mathrm{ng} / \mathrm{ml}]$. We found no association between arterial blood oxygen partial pressure and FGF 21 concentrations. We observed with linear modeling an effect of sample timepoint (F 5.6, $P<0.01)$, poor neurological outcome (F 6.1, $P=0.01$ ), and their interaction (F 3.0, $P=0.03)$, on FGF 21 concentration. In multivariate logistic regression analysis, adjusting for relevant clinical covariates, higher average FGF 21 concentration during the first $72 \mathrm{~h}$ was independently associated with poor neurological outcome (odds ratio 1.60, 95\% confidence interval 1.10-2.32). We conclude that post cardiac arrest patients experience cellular and mitochondrial stress, reflected as a systemic FGF 21 response. This response is higher with a more severe hypoxic injury but it is not exacerbated by hyperoxia.

Abbreviations

FGF 21 Fibroblast growth factor 21

REML Restricted maximum likelihood

Cardiac arrest causes great morbidity, mortality and economic loss ${ }^{1}$. Neurological injury is the most common cause of death during post-resuscitation care ${ }^{2}$ and less than half of those successfully resuscitated survive without neurological sequelae ${ }^{3}$. The cessation of circulation during cardiac arrest causes ischaemia in tissues, disturbing normal cellular function and resulting in cell death. Reperfusion during cardiopulmonary resuscitation and after return of spontaneous circulation (ROSC) leads to generation of reactive oxygen species (ROS) and an inflammatory stress response $e^{4}$. The brain is particularly vulnerable to the hypoxia-reperfusion injury in cardiac arrest due to its high metabolic activity and limited energy reserves ${ }^{5}$, but also extracerebral organ failure complicates

${ }^{1}$ Division of Intensive Care, Department of Anaesthesiology, Intensive Care and Pain Medicine, University of Helsinki and Helsinki University Hospital, PB 340, 00029 Helsinki, Finland. ${ }^{2}$ Department of Emergency Care and Services, University of Helsinki and Helsinki University Hospital, Helsinki, Finland. ${ }^{3}$ Department of Intensive Care, Päijät-Häme Central Hospital, Lahti, Finland. "Department of Neurology, Helsinki University Hospital, Helsinki, Finland. ${ }^{5}$ University of Eastern Finland and Kuopio University Hospital, Kuopio, Finland. ${ }^{\square}$ email: pirkka.pekkarinen@helsinki.fi 
recovery ${ }^{6}$. Several markers of the magnitude of the hypoxia-reperfusion injury and associated inflammation after ROSC and their association with the severity of multiple organ failure have been investigated ${ }^{7-9}$.

Fibroblast growth factor (FGF) 21, an endocrine hormone which may play a role in the development of reperfusion injury after cardiac arrest, is a member of the large FGF family. FGF 21 is derived mainly from the liver, and plays an important role in energy metabolism and homeostasis ${ }^{10}$. Oxidative and inflammatory stress activate the integrated mitochondrial stress response leading to FGF 21 upregulation in the liver and the pancreas, but also in skeletal muscle and adipose tissue ${ }^{11,12}$. Critical illness may cause mitochondrial dysfunction and, accordingly, elevated FGF 21 levels have been reported in intensive care patients ${ }^{13}$. FGF 21 has been shown to ameliorate hypoxia-induced oxidative stress and inflammation in cerebral microvascular endothelial cells ${ }^{14}$ and to protect against myocardial ischaemia-reperfusion injury in a rat model ${ }^{15}$. Accordingly, due to the pivotal role of mitochondria in oxidative metabolism and oxidative stress we tested the hypothesis that FGF 21 would reflect the severity of hypoxia-reperfusion injury after cardiac arrest. Based on previous reports from animal models of brain ischaemia indicating that hyperoxic reperfusion exacerbates mitochondrial dysfunction ${ }^{16}$ and impairs long-term neurological recovery ${ }^{17}$, we also asked, if post-arrest arterial blood oxygen partial pressure $\left(\mathrm{PaO}_{2}\right)$ has an impact on the FGF 21 response.

\section{Methods}

Study setting. This was an observational sub-study of the COMACARE study (NCT02698917). The protocol of the study has been reported elsewhere ${ }^{18,19}$. Briefly, we included unconscious, mechanically ventilated adult (age between 18 and 80 years) Out-of-Hospital Cardiac Arrest (OHCA) patients admitted to the participating ICUs with ventricular fibrillation/tachycardia as initial rhythm and ROSC 10-45 min from the onset of cardiac arrest. All study patients received targeted temperature management of 33 or $36^{\circ} \mathrm{C}$ for $24 \mathrm{~h}$. COMACARE was a $2^{3}$ factorial design study, where patients' treatment targets were randomized to either normoxia $\left[\mathrm{PaO}_{2} 10-15 \mathrm{kPa}\right.$ (75-113 mmHg)] or moderate hyperoxia $\left[\mathrm{PaO}_{2} 20-25 \mathrm{kPa}(150-225 \mathrm{mmHg})\right]$, and high or low normal arterial blood carbon dioxide partial pressure $\left(\mathrm{PaCO}_{2}\right)$ and mean arterial blood pressure (MAP). For the purposes of this study we analyzed data on the patients according to the $\mathrm{PaO}_{2}$ target groups. The study protocol was approved by The Research Ethics Committee of the Northern Savo Hospital District (295/13.02.00/2015\$53). The study was conducted according to the Declaration of Helsinki. Deferred written informed consent was obtained from the next of kin and additionally from all those patients who regained sufficient neurological function for independent decision-making.

Laboratory analysis and control samples. We measured serum FGF 21 with a commercially available sandwich ELISA method (BioVendor Brno, Czech Republic). The detection limit reported by the manufacturer is $7 \mathrm{pg} / \mathrm{ml}$ and calibration range $30 \mathrm{pg} / \mathrm{ml}$ to $1,92 \mathrm{ng} / \mathrm{ml}$. Intra-assay precision is reported as $\mathrm{CV}=2.0 \%$ and interassay precision as $\mathrm{CV}=3.3 \%$. We diluted samples as necessary to receive results within the calibration range. We measured serum FGF 21 concentration from samples collected on ICU admission and 24, 48 and $72 \mathrm{~h}$ after OHCA from 112 patients enrolled in the COMACARE-trial in the six participating Finnish ICUs. We used samples from 40 age- and gender-matched non-critically ill emergency room patients from the largest participating center as controls. This control group was not included in the COMACARE-trial. The controls were participants of another study (NCT03494790), where blood samples from patients with suspected infection were collected in an emergency department. The study protocol including FGF21 analysis was approved in the ethical board (HUS 1423/2017). The controls were chosen before FGF21 analysis and none of them was diagnosed as having had a severe infection.

Outcome. Cerebral Performance Category (CPC) at six months after the cardiac arrest, based on patient records and a telephone interview by an experienced neurologist blinded to the intervention groups, was used as the measure of neurological outcome. We defined 6-month CPC 1-2 as good and CPC 3-5 as poor neurological outcome $\mathrm{o}^{20}$.

Statistics. We assessed group differences with the Mann Whitney U test and Fisher's exact test (continuous and categorial variables, respectively). Correlations between variables were assessed with Spearman's rho. We calculated time-weighted mean $\mathrm{PaO}_{2}$ values for timespans from admission to $24 \mathrm{~h}$ and from 24 to $48 \mathrm{~h}$ of ICU stay by first calculating the mean value for three-hour periods and second the mean of the three-hour means for a 24 -h period. We did this to adjust for the altering frequency in blood gas analysis (for example, poor oxygenation leading to increased frequency of sampling during the first hours of treatment).

We analyzed the association between neurological outcome and temporal change in FGF 21 and the association between $\mathrm{PaO}_{2}$ intervention group and temporal change in FGF 21 using linear models with alternative covariance structures and restricted maximum likelihood (REML) estimation, a method well suited for analyzing longitudinal data with occasional missing values ${ }^{21}$. The MIXED procedure of the SPSS program was used. We tested three potential covariance matrixes, namely, compound symmetry (CS), first order autoregressive (AR1) and unstructured (UN). We chose unstructured covariance matrix for the final analyses because it provided the lowest Akaike's information criterion (AIC) in the presented models. We assessed significant effects and interactions with least-significant-difference tests. The residuals followed a normal distribution on visual inspection in the models, confirming that the assumptions were reasonably met. We used $P<0.05$ as threshold for significance for main effects and interactions and $P<0.0125$ as threshold for significance for the four predefined pairwise comparisons of the interaction of sampling timepoint by outcome.

Finally, we tested the independent predictive value of FGF 21 for neurological outcome in logistic regression models adjusting for confounding variables. To reduce the dynamics of FGF 21 concentration to a single value 


\begin{tabular}{|c|c|c|c|c|}
\hline & $\begin{array}{l}\text { Good outcome } \\
\mathrm{N}=73\end{array}$ & $\begin{array}{l}\text { Poor outcome } \\
\mathrm{N}=39\end{array}$ & $P$-value & $\begin{array}{l}\text { Missing data } \\
\text { Good/poor outcome }\end{array}$ \\
\hline Age (years) & $58(51-66)$ & $66(58-75)$ & $<0.01^{*}$ & $0 / 0$ \\
\hline Sex (male) & $84 \%$ & $80 \%$ & 0.61 & $0 / 0$ \\
\hline $\mathrm{BMI}\left(\mathrm{kg} / \mathrm{m}^{2}\right)$ & $26(24-29)$ & $26(23-29)$ & 0.86 & $0 / 2$ \\
\hline Smoker (yes) & $33 \%$ & $41 \%$ & 0.50 & $6 / 7$ \\
\hline Bystander initiated resuscitation (yes) & $90 \%$ & $69 \%$ & $<0.01^{*}$ & $0 / 0$ \\
\hline Time to first unit $(\mathrm{min})$ & $7.0(6.0-9.0)$ & $7.0(5.0-10)$ & 0.91 & $0 / 0$ \\
\hline Time to $\operatorname{ROSC}^{\mathrm{a}}(\mathrm{min})$ & $17(15-22)$ & $25(22-32)$ & $<0.001^{*}$ & $0 / 0$ \\
\hline APACHE II (point) & $27(24-29)$ & $31(26-35)$ & $<0.01^{*}$ & $0 / 0$ \\
\hline $\mathrm{PaO}_{2}$ group (high) & $47 \%$ & $54 \%$ & 0.55 & $0 / 0$ \\
\hline 48-h highest $\mathrm{PaO}_{2}(\mathrm{kPa})$ & $27(21-32)$ & $29(22-31)$ & 0.64 & $0 / 0$ \\
\hline Time from ICU admission to highest $\mathrm{PaO}_{2}(\mathrm{~h})$ & $2.9(1.9-8.0)$ & $3.7(1.7-10)$ & 0.60 & $0 / 0$ \\
\hline NSE 48 h (ng/ml) & $17(13-25)$ & $49(25-130)$ & $<0.001^{*}$ & $0 / 3$ \\
\hline \multicolumn{5}{|l|}{ FGF 21 (ng/ml) } \\
\hline Admission & $0.66(0.29-1.5)$ & $1.1(0.50-2.3)$ & $0.04^{*}$ & $1 / 0$ \\
\hline $24 \mathrm{~h}$ & $0.92(0.40-1.8)$ & $0.84(0.53-1.7)$ & 0.87 & $0 / 1$ \\
\hline $48 \mathrm{~h}$ & $1.2(0.46-2.3)$ & $1.4(0.43-2.9)$ & 0.46 & $0 / 3$ \\
\hline $72 \mathrm{~h}$ & $0.57(0.19-1.8)$ & $1.2(0.40-2.8)$ & $0.03^{\star}$ & $1 / 5$ \\
\hline
\end{tabular}

Table 1. Characteristics of the study patients $(\mathrm{N}=112)$. Continuous variables: median (IQR), Mann Whitney $\mathrm{U}$ test; categorical variables: percentage of the whole, Fisher's Exact test. Good outcome, 6-month Cerebral Performance Categories (CPC) 1-2; Poor outcome, 6-month CPC 3-5. ${ }^{\star D}$ ifference between groups is statistically significant at the $P<0.05$ level. ${ }^{a}$ ROSC between $10-45 \mathrm{~min}$ was an inclusion criterion for the study.

to be used in the logistic regression models, we calculated the average value of FGF 21 concentration from the available timepoints between admission and $72 \mathrm{~h}$. We tested the following variables as potential confounders in univariate logistic regression: age, sex, body mass index (BMI), current smoker, bystander initiated resuscitation, time from collapse to the arrival of the first unit, time from collapse to ROSC, APACHE II score (excluding age), serum neuron specific enolase (NSE) measured $48 \mathrm{~h}$ after cardiac arrest. Variables with $P<0.3$ in the univariate analyses (age, bystander initiated resuscitation, time to ROSC, APACHE II score excluding age and $48 \mathrm{~h}$ NSE) were selected for the final models. We performed all statistical analyses with the SPSS software (version 24.0, IBM, Armonk, NY, USA). We used GraphPad Prism software, version 8, https://www.graphpad.com to draw the figures.

\section{Results}

Blood samples available for analysis included 111 samples collected at ICU admission [median (IQR) delay from collapse to admission sample was 200 (160-230) min], 111 samples collected at $24 \mathrm{~h}, 109$ samples collected at $48 \mathrm{~h}$ and 106 samples collected at $72 \mathrm{~h}$. Thirty days after cardiac arrest, 36 patients were dead and 76 were alive. The most frequent cause of death was hypoxic ischaemic encephalopathy (32 cases). Six months after cardiac arrest 53 patients had CPC class 1 (good cerebral performance), twenty patients had class 2 (moderate cerebral disability), two patients had class 3 (severe cerebral disability), none had class 4 (vegetative state) and 37 had class 5 (death). Median (interquartile range, IQR) age in forty control patients was 62 (53-69) years and 73\% of them were male. The baseline characteristics of the study population are presented in Table 1.

In the study population the median (IQR) FGF 21 concentration at ICU admission was $0.79(0.37-1.6) \mathrm{ng} / \mathrm{ml}$ and the peak value was $1.2(0.46-2.5) \mathrm{ng} / \mathrm{ml}$ at $48 \mathrm{~h}$. Compared to the ICU admission values, the concentrations in control patients' samples, $0.25(0.094-0.91) \mathrm{ng} / \mathrm{ml}$, were significantly lower $(P<0.001)$. The ICU admission FGF 21 concentration was higher in patients with poor neurological outcome $[1.1(0.50-2.3) \mathrm{ng} / \mathrm{ml}]$ compared to those with a good neurological outcome $[0.66(0.29-1.5) \mathrm{ng} / \mathrm{ml},(P=0.04)]$. Linear correlations between admission FGF 21 concentration and patient age, APACHE II score, time from collapse to ROSC or time from collapse to sampling were not observed (supplementary Figs. 1-4). FGF 21 concentrations at the studied timepoints are presented in Table 1 and Fig. 1.

FGF 21 and oxygen. No significant correlation existed between highest $\mathrm{PaO}_{2}$ value during the first $48 \mathrm{~h}$ of ICU stay and FGF 21 at $48 \mathrm{~h}$ after cardiac arrest (Fig. 2, panel A) or between FGF 21 at $24 \mathrm{~h}$ or $48 \mathrm{~h}$ and mean $\mathrm{PaO}_{2}$ value of the preceding 24-h period (Fig. 2, panel B and C). The distribution of patients with poor and good outcome were comparable over all these scatterplots (Fig. 2).

In the first linear model analysis with REML estimation with FGF 21 concentration as dependent variable, a significant fixed effect of sampling timepoint was found but no effect of $\mathrm{PaO}_{2}$ intervention group or the interaction of time by intervention group on FGF 21 concentration (Table 2).

FGF 21 and neurological outcome. In the second linear model analysis with REML estimation with FGF 21 concentration as dependent variable, there was an effect of sampling timepoint (meaning that FGF 21 


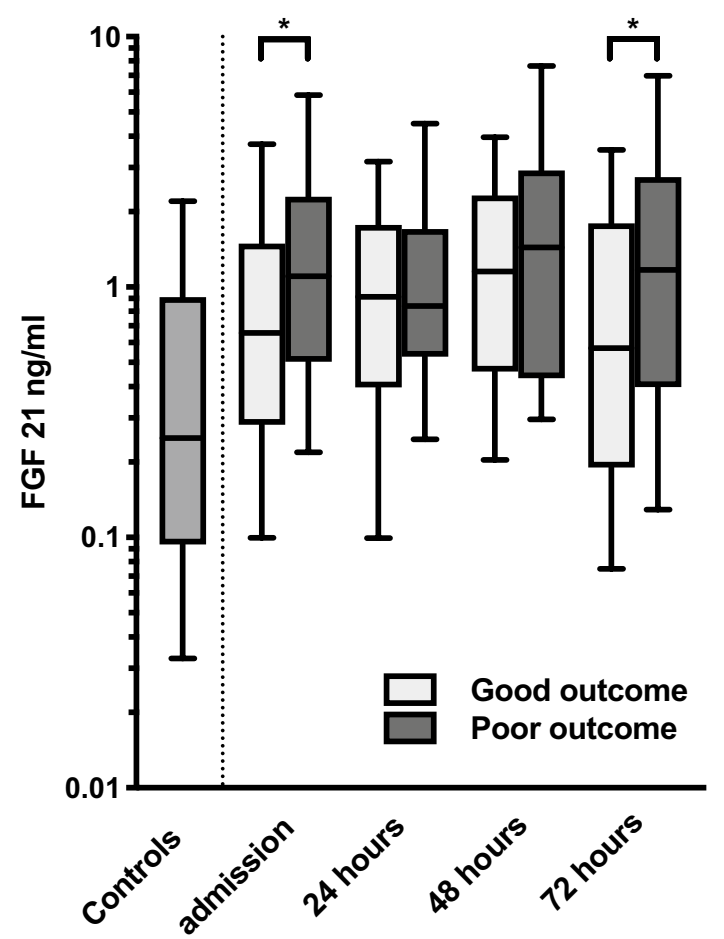

Figure 1. Concentrations of FGF 21 in controls $(\mathrm{N}=40)$ and outcome groups of study patients. Median with interquartile range (box) and 10-90th percentile (whiskers) are presented. Note the logarithmic scale. Good outcome $(\mathrm{N}=73)$, 6-month Cerebral Performance Categories (CPC) 1-2; Poor outcome ( $=39)$, 6-month CPC 3-5. ${ }^{\star}$ Difference between the outcome groups is statistically significant at the $P<0.05$ level (Mann Whitney $\mathrm{U}$ test not adjusted for multiple comparisons).
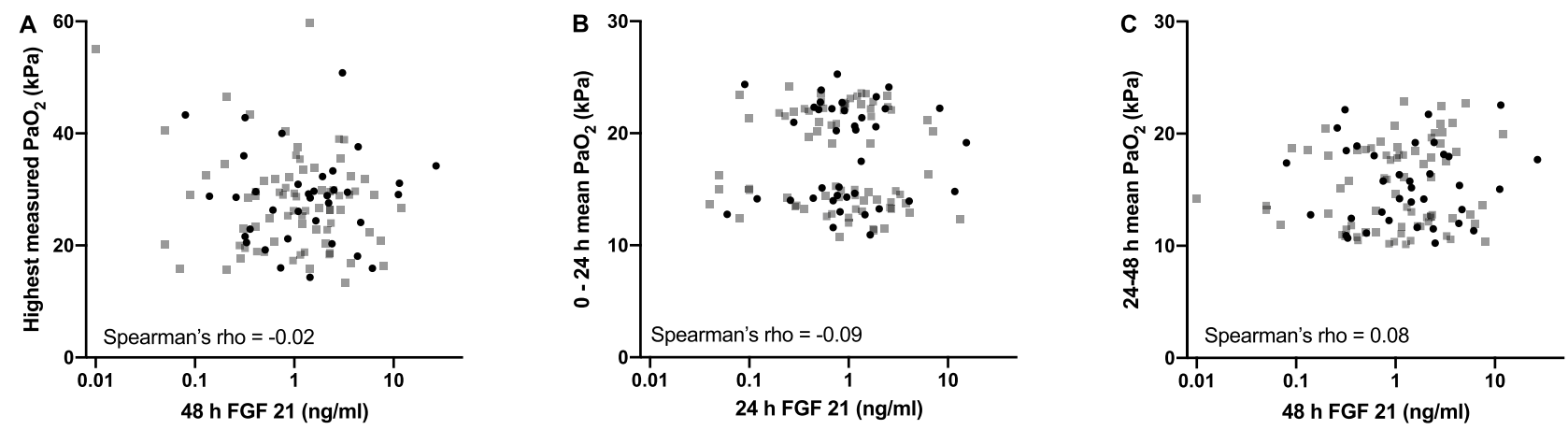

Figure 2. Concentrations of FGF 21 in relation to $\mathrm{PaO}_{2}$. (A) highest arterial blood $\mathrm{PaO}_{2}$ value measured during the first $48 \mathrm{~h}$ of ICU stay and FGF 21 concentration measured at $48 \mathrm{~h}$. (B) time-weighted mean arterial blood $\mathrm{PaO}_{2}$ from admission to $24 \mathrm{~h}$ and FGF 21 concentration measured at $24 \mathrm{~h}$. (C) time-weighted mean arterial blood $\mathrm{PaO}_{2}$ from 24 to $48 \mathrm{~h}$ and FGF 21 concentration measured at $48 \mathrm{~h}$. Black circles, poor outcome; grey squares, good outcome.

\begin{tabular}{|l|l|l|l|}
\hline Fixed effects on FGF 21 concentration $(\mathbf{n}=\mathbf{1 1 2})$ & df & F & $P$-value \\
\hline Timepoint & 3 & 3.9 & $0.01^{*}$ \\
\hline $\mathrm{PaO}_{2}$ group & 1 & 0.09 & 0.77 \\
\hline Timepoint $\times \mathrm{PaO}_{2}$ group & 3 & 1.4 & 0.25 \\
\hline
\end{tabular}

Table 2. Effect of timepoint and $\mathrm{PaO}_{2}$ group. $\mathrm{PaO}_{2}$ group, intervention groups targeting normoxia $\left(\mathrm{PaO}_{2}\right.$ $10-15 \mathrm{kPa})$ or moderate hyperoxia $\left(\mathrm{PaO}_{2} 20-25 \mathrm{kPa}\right)$. ${ }^{*}$ Statistically significant at the $P<0.05$ level. 


\begin{tabular}{|c|c|c|c|}
\hline Fixed effects on FGF 21 concentration $(n=112)$ & df & $\mathbf{F}$ & $P$-value \\
\hline Timepoint & 3 & 5.6 & $<0.01^{*}$ \\
\hline Poor outcome & 1 & 6.1 & $0.01^{*}$ \\
\hline Timepoint $\times$ Poor outcome & 3 & 3.0 & $0.03^{*}$ \\
\hline Pairwise comparisons & Mean difference & $95 \% \mathrm{CI}$ & $P$-value \\
\hline \multicolumn{4}{|l|}{ Timepoint } \\
\hline $24 \mathrm{~h}$-admission & -0.12 & $-0.81-0.58$ & 0.74 \\
\hline $48 \mathrm{~h}-24 \mathrm{~h}$ & 0.83 & $0.42-1.2$ & $<0.001^{\star}$ \\
\hline $72 \mathrm{~h}-48 \mathrm{~h}$ & -0.07 & $-0.92-0.79$ & 0.88 \\
\hline \multicolumn{4}{|l|}{ Poor outcome } \\
\hline Poor-good outcome & 1.2 & $0.24-2.2$ & $0.01^{*}$ \\
\hline \multicolumn{4}{|l|}{ Timepoint $\times$ Poor outcome } \\
\hline $\begin{array}{l}\text { Admission } \\
\text { Poor-good outcome }\end{array}$ & 0.68 & $-0.58-1.9$ & 0.29 \\
\hline $\begin{array}{l}24 \mathrm{~h} \\
\text { Poor-good outcome }\end{array}$ & 0.36 & $-0.61-1.3$ & 0.46 \\
\hline $\begin{array}{l}48 \mathrm{~h} \\
\text { Poor-good outcome }\end{array}$ & 1.4 & $0.002-2.8$ & 0.05 \\
\hline $\begin{array}{l}72 \mathrm{~h} \\
\text { Poor-good outcome }\end{array}$ & 2.5 & $0.62-4.4$ & $<0.01^{\star *}$ \\
\hline
\end{tabular}

Table 3. Effect of timepoint and outcome. Good outcome, 6-month Cerebral Performance Categories (CPC) 1-2; Poor outcome, 6-month CPC 3-5; df, numerator degrees of freedom; CI, confidence interval. * Statistically significant at the $P<0.05$ level (Fixed effects and interactions). ${ }^{*}$ Statistically significant at the $P<0.0125$ level (four pairwise comparisons of interactions).

\begin{tabular}{|l|l|l|c|}
\hline & OR & $\mathbf{9 5 \%}$ CI & P-value \\
\hline Age (year) & 1.09 & $1.01-1.17$ & $0.03^{*}$ \\
\hline Bystander initiated resuscitation (no) & 11.5 & $1.99-66.5$ & $<0.01^{*}$ \\
\hline Delay to ROSC (min) & 1.15 & $1.04-1.26$ & $<0.01^{*}$ \\
\hline APACHE II (excluding age, point) & 0.99 & $0.84-1.18$ & 0.91 \\
\hline NSE 48 h (ng/ml) & 1.11 & $1.05-1.19$ & $<0.01^{*}$ \\
\hline FGF 21 mean concentration admission to $72 \mathrm{~h}(\mathrm{ng} / \mathrm{ml})$ & 1.60 & $1.10-2.32$ & $0.01^{*}$ \\
\hline
\end{tabular}

Table 4. Logistic regression model for predicting 6-month poor neurological outcome (CPC 3-5). Good outcome, 6-month Cerebral Performance Categories (CPC) 1-2 (neurological condition ranging from "good cerebral performance" to "moderate cerebral disability"); Poor outcome, 6-month CPC 3-5 (neurological condition ranging from "severe cerebral disability" to "death"); OR, odds ratio; CI, confidence interval. * Statistically significant at the $P<0.05$ level.

concentrations were significantly different between timepoints) and of poor neurological outcome (meaning that FGF 21 concentrations were significantly different between outcome groups) and an interaction of sampling timepoint with poor neurological outcome (meaning that the dynamics of FGF 21 concentration were significantly different between outcome groups) (Table 3). Pairwise comparison of estimated marginal means at different timepoints indicated a significant increase between 24 and $48 \mathrm{~h}$ (mean difference $0.83 \mathrm{ng} / \mathrm{ml}, 95 \% \mathrm{CI}$ $0.42-1.2 \mathrm{ng} / \mathrm{ml}, P<0.001$, Table 3 ). The estimated marginal means were higher in the poor outcome group (mean difference $1.2 \mathrm{ng} / \mathrm{ml}, 95 \%$ CI $0.24-2.2 \mathrm{ng} / \mathrm{ml}, P=0.01$, Table 3). Pairwise comparisons of the means defined by sampling timepoint by outcome group interaction, indicated a statistically significant difference at the $P<0.0125$ level at $72 \mathrm{~h}$ timepoint (mean difference $2.5 \mathrm{ng} / \mathrm{ml}, 95 \%$ CI $0.62-4.4 \mathrm{ng} / \mathrm{ml}, P<0.01$, Table 3 ).

In a logistic regression model adjusting for relevant confounding variables, average FGF 21 concentration during the first $72 \mathrm{~h}$ after cardiac arrest was independently associated with poor 6-month neurological outcome (CPC 3-5) (Table 4). The results were similar when the logistic regression model was replicated for predicting any 6-month neurological status worse than "good cerebral performance" (i.e. CPC 2-5) (Table 5).

\section{Discussion}

The main finding of this study is that serum FGF 21 levels were significantly elevated after successful resuscitation in ICU-treated OHCA patients with shockable initial rhythm. Furthermore, the temporal pattern of FGF 21 concentration during the first $72 \mathrm{~h}$ was different in patients with poor and good 6-month neurological outcome. We found no association between arterial blood oxygen partial pressures $\left(\mathrm{PaO}_{2}\right)$ and the FGF 21 concentrations in patients after cardiac arrest. 


\begin{tabular}{|l|l|l|c|}
\hline & OR & 95\% CI & P-value \\
\hline Age (year) & 1.09 & $1.03-1.16$ & $<0.01^{*}$ \\
\hline Bystander initiated resuscitation (no) & 2.31 & $0.51-10.5$ & 0.28 \\
\hline Delay to ROSC (min) & 1.16 & $1.06-1.26$ & $<0.01^{*}$ \\
\hline APACHE II (excluding age, point) & 1.01 & $0.90-1.14$ & 0.86 \\
\hline NSE $48 \mathrm{~h}(\mathrm{ng} / \mathrm{ml})$ & 1.07 & $1.01-1.13$ & $0.02^{*}$ \\
\hline FGF $21 \mathrm{mean}$ concentration admission to $72 \mathrm{~h}(\mathrm{ng} / \mathrm{ml})$ & 1.50 & $1.11-2.02$ & $<0.01^{*}$ \\
\hline
\end{tabular}

Table 5. Logistic regression model for predicting 6-month Cerebral Performance Categories 2-5 (neurological condition not classified as CPC 1 "good cerebral performance"). OR, odds ratio; CI, confidence interval. * Statistically significant at the $P<0.05$ level.

The ischaemia-reperfusion injury associated with cardiac arrest is often followed by an inflammatory reaction resembling septic shock ${ }^{22}$, which may lead to multiple organ failure and death in cardiac arrest patients. In our patient cohort we observed elevated FGF 21 levels on ICU admission and an increase between 24 and 48 h timepoints. Interestingly, FGF 21 has been shown to play an anti-inflammatory role in sepsis ${ }^{23}$ and hypoxia-induced pulmonary hypertension ${ }^{24}$ and to protect myocardium ${ }^{25}$ and neurons ${ }^{26}$ in oxidative stress. Therefore, increasing FGF 21 levels can be interpreted to reflect a protective response to severe perturbations in oxidative metabolism.

Our findings are in line with previous data reporting elevated FGF 21 levels in general population of critical care patients ${ }^{13}$. The emerging role of FGF 21 as a marker of mitochondrial dysfunction ${ }^{27}$ combined with reports of elevated FGF 21 predicting cardiovascular events ${ }^{28-30}$ make cardiac arrest an especially interesting condition for studying FGF 21 due to the extreme ischaemia-reperfusion event of circulatory standstill followed by resuscitation.

Transcription of FGF 21 is regulated by multiple pathways ${ }^{31}$. Disturbance of mitochondrial function leads to upregulation of FGF 21 transcription by ATF $4^{32}$, which is a central player in the integrated stress response signaling network ${ }^{13,33}$. Lactate induces FGF 21 expression in a p38-MAPK dependent manner ${ }^{34}$ and activation of glucocorticoid receptors increases FGF 21 expression ${ }^{35}$. Expression of mRNA in tissues and systemic FGF 21 protein levels change rapidly in response to stressful stimuli. In liver transplants, a robust increase in the expression of FGF 21 in hepatocytes has been reported with peak systemic concentrations in the recipient as early as $2 \mathrm{~h}$ after return of hepatic blood flow ${ }^{36}$. In cardiac surgery and ST-elevation myocardial infarction, peak systemic concentrations of FGF 21 were reached $6 \mathrm{~h}$ after the insult ${ }^{37,38}$. After a short stressful insult such as cardiac surgery or liver transplantation, systemic FGF 21 returns to basal level in a few days ${ }^{36,37}$, whereas FGF 21 levels in ICU patients remain elevated during the length of the ICU stay ${ }^{13}$.

Based on these data, it is plausible that the peak systemic concentrations of FGF 21 observed at $48 \mathrm{~h}$ in the current study are a result of induced transcription in the liver and other tissues in response to hypoxia-reperfusion injury associated with the cardiac arrest. The difference in admission values of FGF 21 between outcome groups (Table 1, Fig. 1) suggests faster elevation in the poor outcome group, although it may also partly reflect the cardiovascular risk factors preceding the cardiac arrest ${ }^{28-30}$. The association of higher FGF 21 concentrations during the first $72 \mathrm{~h}$ with poor 6-month outcome (Tables 3, 4, 5) suggest that the stressful process leading to FGF 21 expression fails to resolve in the poor outcome group. In line with this, a downward trend in the median FGF 21 concentration between 48 and $72 \mathrm{~h}$ can be seen in the good but not in the poor outcome group (Fig. 1).

Therapeutic hypothermia may have an impact on FGF 21 expression ${ }^{38}$. However, admission samples collected already before cooling had increased FGF 21 levels (Table 1, Fig. 1) and although part of the observed dynamics of FGF 21 after cardiac arrest may be associated with hypothermia, this cannot explain the differences seen between outcome groups (Tables 3, 4, 5) since all study patients received therapeutic hypothermia.

Global tissue hypoxia is considered a central pathophysiological trigger for the post cardiac arrest syndrome ${ }^{39}$. However, after reperfusion of the organs, excess oxygen can also be detrimental. In experimental conditions hyperoxemia during post-resuscitation period has been reported to exacerbate reperfusion injury ${ }^{16,17}$, and retrospective studies of clinical data have suggested that even short exposure to hyperoxia may be associated with mortality in cardiac arrest patients ${ }^{40,41}$. However, our group has been unable to replicate this result in a prospective observational study ${ }^{42}$ and a large registry study ${ }^{43}$. If this association would hold true, mitochondrial injury induced by increased reactive oxygen species production stimulated by hyperoxia would provide a plausible mechanistic explanation. In the current study we found no association between either highest measured $\mathrm{PaO}_{2}$ value or preceding 24-h mean $\mathrm{PaO}_{2}$ values and FGF 21 concentration as a surrogate marker for mitochondrial stress (Fig. 2).

As discussed above, FGF 21 appears to protect against damaging inflammation, reperfusion injury and oxidative stress when administered to cell cultures or laboratory animals ${ }^{23-26}$. This protective measure can be overwhelmed, and FGF 21 has been shown to predict renal injury after coronary angiography ${ }^{44}$, liver failure in critically ill patients with cirrhosis ${ }^{45}$, and poor outcome in patients with sepsis ${ }^{46,47}$. To the best of our knowledge, there are no previous animal or human studies assessing the FGF 21 response following cardiac arrest or its association with post-resuscitation neurological outcome. In the current study, higher serum FGF 21 concentrations during post-resuscitation care were associated with 6-month poor neurological outcome (Tables 3, 4, 5). The magnitude of the FGF 21 response appears to reflect the severity of the systemic hypoxia-reperfusion injury in cardiac arrest patients, although the large variation in FGF 21 concentrations does not allow its use for prognostication on single patient level. 


\section{Strengths and limitations of the study}

This was a multicenter study conducted in a government-funded healthcare system with good research infrastructure. No patient was lost to follow-up and sera were available for all four studied timepoints. The assessor of outcome was blinded and FGF 21 measurements were performed blinded to clinical information. Our study has also limitations. First, the patient population consisted of only OHCA patients with VF/VT as the initial cardiac rhythm and, thus, the generalizability of our findings regarding all cardiac arrest patients is limited. Second, the median time for the first unit to reach the patient was only $7 \mathrm{~min}$, which is not the case in less urban patient cohorts. Third, we had no data on prehospital $\mathrm{PaO}_{2}$ or on the pre-arrest FGF 21 levels. Since elevated FGF 21 levels are associated with increased risk of cardiovascular events, there is a possibility that elevated FGF 21 levels after cardiac arrest partly reflect the pre-arrest chronic morbidity of the cardiovascular system and not only conditions associated with the acute event. Finally, this was a rather small pilot study with only 39 poor outcome events, limiting the statistical power of the study.

\section{Conclusions}

Our results suggest a role for FGF 21 in the hypoxia-reperfusion injury following cardiac arrest. Critically ill patients resuscitated after OHCA have elevated FGF 21 concentrations on ICU admission and the temporal pattern and magnitude of FGF 21 response seem to differ according to 6-month neurological outcome. The FGF 21 response is not affected by moderate hyperoxia during ICU care. The large variation on FGF 21 concentrations discourage its clinical use for outcome prediction in cardiac arrest.

\section{Data availability}

The dataset analyzed during the current study includes sensitive patient information. Legal restrictions prohibit us from making the data publicly available. Data excluding patient identifying information are available from the corresponding author on reasonable request.

Received: 7 July 2020; Accepted: 15 December 2020

Published online: 12 January 2021

\section{References}

1. Efendijev, I. et al. Outcomes and healthcare-associated costs one year after intensive care-treated cardiac arrest. Resuscitation 131, 128-134. https://doi.org/10.1016/j.resuscitation.2018.06.028 (2018).

2. Lemiale, V. et al. Intensive care unit mortality after cardiac arrest: the relative contribution of shock and brain injury in a large cohort. Intensive Care Med. 39, 1972-1980. https://doi.org/10.1007/s00134-013-3043-4 (2013).

3. Sasson, C., Rogers, M. A., Dahl, J. \& Kellermann, A. L. Predictors of survival from out-of-hospital cardiac arrest: a systematic review and meta-analysis. Circ. Cardiovasc. Qual. Outcomes 3, 63-81. https://doi.org/10.1161/circoutcomes.109.889576 (2010).

4. Welbourn, C. \& Efstathiou, N. How does the length of cardiopulmonary resuscitation affect brain damage in patients surviving cardiac arrest? A systematic review. Scand. J. Trauma Resuscitation Emerg. Med. 26, 77. https://doi.org/10.1186/s13049-018-0476-3 (2018).

5. Dragancea, I., Rundgren, M., Englund, E., Friberg, H. \& Cronberg, T. The influence of induced hypothermia and delayed prognostication on the mode of death after cardiac arrest. Resuscitation 84, 337-342. https://doi.org/10.1016/j.resuscitation.2012.09.015 (2013).

6. Pekkarinen, P. T. et al. Association of extracerebral organ failure with 1-year survival and healthcare-associated costs after cardiac arrest: an observational database study. Crit. Care 23, 67. https://doi.org/10.1186/s13054-019-2359-z (2019).

7. Ristagno, G. et al. Elevations of inflammatory markers ptx 3 and sst 2 after resuscitation from cardiac arrest are associated with multiple organ dysfunction syndrome and early death. Clin. Chem. Lab. Med. 53, 1847-1857. https://doi.org/10.1515/cclm-20141271 (2015).

8. Pekkarinen, P. T. et al. Procalcitonin and presepsin as prognostic markers after out-of-hospital cardiac arrest. Shock 50, 395-400. https://doi.org/10.1097/SHK.0000000000001087 (2018).

9. Vaahersalo, J. et al. Admission interleukin-6 is associated with post resuscitation organ dysfunction and predicts long-term neurological outcome after out-of-hospital ventricular fibrillation. Resuscitation 85, 1573-1579. https://doi.org/10.1016/j.resuscitat ion.2014.08.036 (2014).

10. BonDurant, L. D. \& Potthoff, M. J. Fibroblast growth factor 21: a versatile regulator of metabolic homeostasis. Annu Rev. Nutr. 38, 173-196. https://doi.org/10.1146/annurev-nutr-071816-064800 (2018).

11. Luo, Y. et al. Rush to the fire: Fgf21 extinguishes metabolic stress, metaflammation and tissue damage. Cytokine Growth Factor Rev. 38, 59-65. https://doi.org/10.1016/j.cytogfr.2017.08.001 (2017).

12. Khan, N. A. et al. Mtorc1 regulates mitochondrial integrated stress response and mitochondrial myopathy progression. Cell Metab. 26, 419-428.e415. https://doi.org/10.1016/j.cmet.2017.07.007 (2017).

13. Thiessen, S. E., Vanhorebeek, I., Derese, I., Gunst, J. \& Van den Berghe, G. Fgf21 response to critical illness: Effect of blood glucose control and relation with cellular stress and survival. J. Clin. Endocrinol. Metab. 100, E1319-1327. https://doi.org/10.1210/jc.20152700 (2015).

14. Wang, H. W. et al. Fgf21 protects against hypoxia injury through inducing hsp72 in cerebral microvascular endothelial cells. Front Pharmacol. 10, 101. https://doi.org/10.3389/fphar.2019.00101 (2019).

15. Hu, S., Cao, S., Tong, Z. \& Liu, J. Fgf21 protects myocardial ischemia-reperfusion injury through reduction of mir-145-mediated autophagy. Am. J. Transl. Res. 10, 3677-3688 (2018).

16. Fiskum, G. et al. Postischemic oxidative stress promotes mitochondrial metabolic failure in neurons and astrocytes. Ann. N. Y. Acad. Sci. 1147, 129-138. https://doi.org/10.1196/annals.1427.026 (2008).

17. Hazelton, J. L. et al. Hyperoxic reperfusion after global cerebral ischemia promotes inflammation and long-term hippocampal neuronal death. J. Neurotrauma 27, 753-762. https://doi.org/10.1089/neu.2009.1186 (2010).

18. Jakkula, P. et al. Targeting low- or high-normal carbon dioxide, oxygen, and mean arterial pressure after cardiac arrest and resuscitation: study protocol for a randomized pilot trial. Trials 18, 507. https://doi.org/10.1186/s13063-017-2257-0 (2017).

19. Jakkula, P. et al. Targeting two different levels of both arterial carbon dioxide and arterial oxygen after cardiac arrest and resuscitation: A randomised pilot trial. Intensive Care Med. https://doi.org/10.1007/s00134-018-5453-9 (2018).

20. Cummins, R. O. et al. Recommended guidelines for uniform reporting of data from out-of-hospital cardiac arrest: the utstein style. A statement for health professionals from a task force of the American Heart Association, the European Resuscitation Council, the Heart and Stroke Foundation of Canada, and the Australian Resuscitation council. Circulation 84, 960-975 (1991). 
21. Duricki, D. A., Soleman, S. \& Moon, L. D. Analysis of longitudinal data from animals with missing values using spss. Nat. Protoc. 11, 1112-1129. https://doi.org/10.1038/nprot.2016.048 (2016).

22. Adrie, C. et al. Successful cardiopulmonary resuscitation after cardiac arrest as a "sepsis-like" syndrome. Circulation 106, 562-568. https://doi.org/10.1161/01.CIR.0000023891.80661.AD (2002).

23. Li, J. Y. et al. Fgf-21 elevated il-10 production to correct lps-induced inflammation. Inflammation 41, 751-759. https://doi. org/10.1007/s10753-018-0729-3 (2018).

24. Liu, J. et al. Fibroblast growth factor 21 attenuates hypoxia-induced pulmonary hypertension by upregulating ppargamma expression and suppressing inflammatory cytokine levels. Biochem. Biophys. Res. Commun. 504, 478-484. https://doi.org/10.1016/j. bbrc.2018.09.004 (2018).

25. Planavila, A. et al. Fibroblast growth factor 21 protects the heart from oxidative stress. Cardiovasc. Res. 106, 19-31. https://doi. org/10.1093/cvr/cvu263 (2015).

26. Lu, Y. et al. Fibroblast growth factor 21 facilitates peripheral nerve regeneration through suppressing oxidative damage and autophagic cell death. J. Cell Mol. Med. https://doi.org/10.1111/jcmm.13952 (2018).

27. Morovat, A. et al. Use of fgf- 21 as a biomarker of mitochondrial disease in clinical practice. J. Clin. Med. https://doi.org/10.3390/ jcm6080080 (2017).

28. Ong, K. L. et al. High plasma fgf 21 levels predicts major cardiovascular events in patients treated with atorvastatin (from the treating to new targets [tnt] study). Metab. Clin. Exp. https://doi.org/10.1016/j.metabol.2018.11.006 (2018).

29. Cheng, J., Su, X., Qiao, L., Zhai, C. \& Chen, W. Circulating level of fibroblast growth factor 21 is independently associated with the risks of unstable angina pectoris. Biosci. Rep. https://doi.org/10.1042/BSR20181099 (2018).

30. Zhang, W., Chu, S., Ding, W. \& Wang, F. Serum level of fibroblast growth factor 21 is independently associated with acute myocardial infarction. PLoS ONE 10, e0129791. https://doi.org/10.1371/journal.pone.0129791 (2015).

31. Bae, K. H., Kim, J. G. \& Park, K. G. Transcriptional regulation of fibroblast growth factor 21 expression. Endocrinol. Metab. (Seoul) 29, 105-111. https://doi.org/10.3803/EnM.2014.29.2.105 (2014).

32. Kim, K. H. et al. Autophagy deficiency leads to protection from obesity and insulin resistance by inducing fgf 21 as a mitokine. Nat. Med. 19, 83-92. https://doi.org/10.1038/nm.3014 (2013).

33. Costa-Mattioli, M. \& Walter, P. The integrated stress response: from mechanism to disease. Science https://doi.org/10.1126/scien ce.aat5314 (2020).

34. Jeanson, Y. et al. Lactate induces fgf 21 expression in adipocytes through a p38-mapk pathway. Biochem. J. 473, 685-692. https:// doi.org/10.1042/bj20150808 (2016).

35. Patel, R. et al. Glucocorticoids regulate the metabolic hormone fgf21 in a feed-forward loop. Mol. Endocrinol. 29, 213-223. https ://doi.org/10.1210/me.2014-1259 (2015).

36. Ye, D. et al. Circulating fibroblast growth factor 21 is a sensitive biomarker for severe ischemia/reperfusion injury in patients with liver transplantation. Sci. Rep. 6, 19776. https://doi.org/10.1038/srep19776 (2016).

37. Kotulak, T. et al. Increased circulating and epicardial adipose tissue mrna expression of fibroblast growth factor- 21 after cardiac surgery: possible role in postoperative inflammatory response and insulin resistance. Physiol. Res. 60, 757-767 (2011).

38. Mohammad, M. A. et al. Proteomics in hypothermia as adjunctive therapy in patients with st-segment elevation myocardial infarction: a chill-mi substudy. Therap. Hypotherm. Temp. Manag. 7, 152-161. https://doi.org/10.1089/ther.2016.0041 (2017).

39. Nolan, J. P. et al. Post-cardiac arrest syndrome: Epidemiology, pathophysiology, treatment, and prognostication. A scientific statement from the international liaison committee on resuscitation; The American Heart Association Emergency Cardiovascular Care Committee; The Council on Cardiovascular Surgery and Anesthesia; The Council on Cardiopulmonary, Perioperative, and Critical Care; The Council on Clinical Cardiology; The Council on Stroke. Resuscitation 79, 350-379. https://doi.org/10.1016/j.resuscitat ion.2008.09.017 (2008).

40. Elmer, J. et al. The association between hyperoxia and patient outcomes after cardiac arrest: analysis of a high-resolution database. Intensive Care Med. 41, 49-57. https://doi.org/10.1007/s00134-014-3555-6 (2015).

41. Kilgannon, J. H. et al. Relationship between supranormal oxygen tension and outcome after resuscitation from cardiac arrest. Circulation 123, 2717-2722. https://doi.org/10.1161/circulationaha.110.001016 (2011).

42. Vaahersalo, J. et al. Arterial blood gas tensions after resuscitation from out-of-hospital cardiac arrest: associations with long-term neurologic outcome. Crit. Care Med. 42, 1463-1470. https://doi.org/10.1097/ccm.0000000000000228 (2014).

43. Humaloja, J. et al. Early hyperoxemia is not associated with cardiac arrest outcome. Resuscitation 140, 185-193. https://doi. org/10.1016/j.resuscitation.2019.04.035 (2019).

44. Wu, C. H. et al. Circulating fibroblast growth factor 21 is associated with subsequent renal injury events in patients undergoing coronary angiography. Sci. Rep. 8, 12425. https://doi.org/10.1038/s41598-018-30744-8 (2018).

45. Ruiz-Margain, A. et al. Fibroblast growth factor 21 is an early predictor of acute-on-chronic liver failure in critically ill patients with cirrhosis. Liver Transplant. Off. Publ. Am. Assoc. Study Liver Dis. Int. Liver Transplant. Soc. 24, 595-605. https://doi.org/10.1002/ lt.25041 (2018).

46. $\mathrm{Li}$, X. et al. Predictive value of combined serum fgf 21 and free $\mathrm{t} 3$ for survival in septic patients. Clin. Chim. Acta Int. J. Clin. Chem. 494, 31-37. https://doi.org/10.1016/j.cca.2019.03.005 (2019).

47. Li, X. et al. Early increases in serum fgf21 levels predict mortality of septic patients. Cytokine https://doi.org/10.1016/j. cyto.2018.05.020 (2018).

\section{Acknowledgments}

The authors thank prof. Anu Wartiovaara for providing laboratory facilities and Tuomas Selander for guidance with the statistical analyses. The great collaborative efforts of all participants of the COMACARE study are acknowledged.

\section{Author contributions}

P.T.P. and J.H. planned the study and wrote the manuscript with M.B.S, V.L. and M.R.; P.T.P. performed the laboratory- and statistical analyses and drew the figures. M.B.S., P.J., L.A., P.L., M.T., V.P., M.R. and J.H. participated in conducting the COMACARE study. All authors read and revised the manuscript for substantial intellectual content and approved submission.

\section{Funding}

The study was supported by a competitive research grant from Orion Research Foundation sr., and Helsinki University Hospital funding (project M7100YLIT2) to P.T.P.; Helsinki University Hospital (state funding: Y102011095) to J.H. 


\section{Competing interests}

The authors declare no competing interests.

\section{Additional information}

Supplementary Information The online version contains supplementary material available at https://doi. org/10.1038/s41598-020-80086-7.

Correspondence and requests for materials should be addressed to P.T.P.

Reprints and permissions information is available at www.nature.com/reprints.

Publisher's note Springer Nature remains neutral with regard to jurisdictional claims in published maps and institutional affiliations.

(c) (1) Open Access This article is licensed under a Creative Commons Attribution 4.0 International License, which permits use, sharing, adaptation, distribution and reproduction in any medium or format, as long as you give appropriate credit to the original author(s) and the source, provide a link to the Creative Commons licence, and indicate if changes were made. The images or other third party material in this article are included in the article's Creative Commons licence, unless indicated otherwise in a credit line to the material. If material is not included in the article's Creative Commons licence and your intended use is not permitted by statutory regulation or exceeds the permitted use, you will need to obtain permission directly from the copyright holder. To view a copy of this licence, visit http://creativecommons.org/licenses/by/4.0/.

(C) The Author(s) 2021 